\title{
EVOLUCIÓN DE UN SECTOR COSTERO DURANTE LA ALTA EDAD MEDIA EN EL NW DE LA PENÍNSULA IBÉRICA
}

\author{
REBECA TALLÓN ARMADA ${ }^{(1)}$, MANUELA COSTA-CASAIS ${ }^{(2)} \&$ TERESA TABOADA RODRÍGUEZ $^{(1)}$
}

Resumen:

\begin{abstract}
En el presente trabajo se estudia la evolución de un tramo costero de la Ría de Vigo (NW de la península Ibérica) desde época romana hasta el siglo XVII, prestando particular atención a la Alta Edad Media. Desde el punto de vista arqueológico, la importancia del sector radica en la presencia de una salina de época romana, en uso desde los siglos II BC a III-IV AD, y su abandono para dar paso a la instauración de una iglesia y una necrópolis en la Alta Edad Media (a partir de los siglos IV-V AD). Se muestrearon, con alta resolución, tres secuencias edafo-sedimentarias y se analizaron diversas propiedades físico-químicas $(\mathrm{pH}$, granulometría, contenido en $\mathrm{C}, \mathrm{N}, \mathrm{S}, \mathrm{P}, \mathrm{Fe}, \mathrm{As}$ ) y la composición mineralógica, con el fin de determinar la naturaleza de las distintas facies presentes, los procesos implicados y las causas asociadas. Los resultados indican modificaciones en la costa entre finales del periodo romano y comienzos de la Alta Edad Media, con una evolución del medio desde una dinámica costera, con formaciones de marisma-lagoon costero, a una dinámica eólica, con dunas. Dicho cambio es coincidente con el cese de la explotación romana de sal y el inicio del periodo Frío Altomedieval. Estos resultados son similares a los encontrados en otros puntos de la Ría de Vigo. En los tres sectores comparados (Rosalía de Castro, Toralla y Hospital), a la par que se produce la progradación de las formaciones de marisma y duna, el uso antrópico del sector cambia. Aunque la pauta climática presenta un papel importante en la evolución de este sector, los cambios en la línea de costa que se detectan entre ambos periodos culturales pudo estar determinada también por causas socioecómicas.
\end{abstract}

Palabras clave: pedoarqueología, evolución costera, Alta Edad Media, NW Iberia

Abstract:

Evolution of a coastal area during the early Middle Ages in the NW of the Iberian Peninsula.

This paper focuses on the evolution of a coastal stretch of the Ria de Vigo (NW Iberian Peninsula) during the early Middle Ages, which presents an important archaeological site with a roman salt mine. Salt exploitation in the Roman period ceased around the AD 3rd-5th centuries, and was followed by the establishment of a churche and a cemetery during the Middle Ages. The properties ( $\mathrm{pH}$, grain size, $\mathrm{C}, \mathrm{N}, \mathrm{S}, \mathrm{P}, \mathrm{Fe}$ and As, and mineralogical composition) of three pedo-sedimentary sequences were analysed in order to identify the different environmental changes that affected this sector. The results suggest signifcant changes occurred in the coast from the late Roman period ( $\mathrm{AD}$ 3rth-5th centuries) into the early Middle Ages ( $\mathrm{AD}$ 5th-6th centuries), with an evolution from a marine-continental dynamics, with formation of salt marshes, to a wind dynamics, with dune formations. This change is consistent with the abandonment of the Roman salt exploitation and the beginning of Cold Dark Ages. These results are similar to those found in other areas of the Ría de Vigo. In the three sectors compared (Rosalia de Castro, Toralla and Hospital) land use changed while the progradation of marsh and dune formations occurred. Although climate may have played a major role in the evolution of this sector, the changes in the coastline between the two cultural periods could be also related to socio-economic causes.

Keywords: edoarchaeology, coastal evolution, Early Middle Age, NW Iberia

Received: 16 December, 2014; Accepted: 28 April, 2015

\section{INTRODUCCIÓN}

El litoral es una zona de interacción entre el mar y el continente que ofrece gran variedad de ambientes y ecosistemas y, por lo tanto, numerosos recursos, tanto desde el punto de vista físico como biológico, convirtiéndose por esto desde época muy temprana en una importante fuente de alimentación y un enclave primordial para la conexión y comercio entre distintos lugares y culturas. A su vez, se caracteriza por ser un medio de interfaz, en el que confluyen procesos continentales y marinos, lo que genera una dinámica muy activa que favorece el constante cambio del paisaje costero tanto a micro como a macro escala (CARTER 1988; CARTER \& WOODROFFE 1994).

Los principales cambios que dejan su huella en las formaciones superficiales litorales, están relacionados con variaciones en los factores que controlan su dinámica (las mareas, corrientes, oleaje, vientos, temporales, escorrentía superficial, erosión de suelos, modificaciones en la red de drenaje, etc...), debido tanto a causas de origen ambiental como antrópicas. Entre las primeras, las más relevantes son las variaciones del nivel del mar en respuesta a

(1) Departamento de Edafoloxía e Química Agrícola, Facultade de Bioloxía, Universidade de Santiago de Compostela Rua López Gómez de Marzoa, s/n, Campus Sur, 15706. Santiago de Compostela, A Coruña, España. Email: rebeca.tallon@usc.es; teresa.taboada@usc.es

${ }^{(2)}$ Departamento de Xeografia, Facultade de Xeografia e Historia, Universidade de Santiago de Compostela, Praza da Universidade 1, 15782, Santiago de Compostela, A Coruña, España. Email: manuela.costa@usc.es 
cambios climáticos y la consiguiente reconfiguración de la línea de costa (MARTínEZ CORTIZAS \& COSTA CASAIS 1997), y entre las segundas la deforestación, la erosión de suelos o la reestructuración del espacio como resultado de los asentamientos humanos (MARTínEZCORTIZAS et al. 2009).

Dentro de las formaciones superficiales litorales, las secuencias edafo-sedimentarias costeras aportan una valiosa información sobre la evolución del litoral al tener una alta capacidad para registrar los cambios que ocurren en el medio, reflejarlos en sus propiedades y que prevalezcan en el tiempo. Esto se observa en las distintas investigaciones que emplean este tipo de secuencias para el estudio de la evolución de sectores costeros del NW peninsular (GRANJA et al. 1999, 2008, 2010; MEIRELES \& TEXIER 2000; DIAS et al. 2002; ABRANTES et al. 2005; DE CARVALHO et al. 2006; ARAÚJO 2008; BAPTISTA et al. 2014; CARVALHIDO et al. 2014), estando una gran parte de estos trabajos centrados en la costa de Galicia (FÁBREGAS et al. 2003; ALONSO \& PAGÉs 2007; BAO et al. 2007; BLANCO-CHAO et al. 2007; COSTA CASAIS et al. 2007-2008; ARAÚJO 2008; ANDRADE et al., 2014). Su configuración marcada por las numerosas rías, ofrece una alta variabilidad morfológica, con multitud de entrantes y salientes que favorecen las zonas de acumulación y permiten que las secuencias edafo-sedimentarias sean abundantes y con buena resolución, estos depósitos presentan una amplia distribución espacial con evidencias de presión antrópicas diferentes. (COSTA CASAIS 2001; GOMEZ-GESTEIRA et al. 2011).

En este trabajo nos aproximamos a la evolución de un tramo de la costa de Galicia, entre los siglos VX (Alta Edad Media), en relación a los cambios ambientales y la actividad humana. El área de estudio se encuentra en el margen sur de la Ría de Vigo, al pie de la ciudad de Vigo, y cuenta con el valor añadido de estar en un contexto arqueológico de explotación de sal en época romana. Dicho sector se encuentra en el núcleo de la industria conservera que los romanos crean en torno a las Rías Baixas, a través de distintas salinas y factorías de pescado instauradas por toda la Ría de Vigo, organizadas y gestionadas desde el centro portuario romano de Vigo (CURRÁs 2007). Desde el punto de vista paleoambiental, este sector presenta un registro edafo-sedimentario prácticamente continuo, con un rango cronológico de unos 4000 años, cuya caracterización general está descrita en trabajos previos (TALLÓN ARMADA 2012; TALLÓN ARMADA et al. 2013; SILVA SÁNCHEZ et al. 2013).

El objetivo principal de nuestro estudio es la reconstrucción paleoambiental de este tramo costero a partir del análisis sedimentológico, edáfico, geoquímico y mineralógico de secuencias edafo-sedimentarias, para conocer su evolución durante el periodo de la Alta Edad Media, comparando los resultados con los periodos culturales precedente y posterior.

\section{MATERIAL Y MÉTODOS}

\section{1 Área de estudio}

El área de estudio abarca un yacimiento de explotación de sal de época romana definido como Parcela P3-6 y ubicado en la calle Rosalía de Castro (Vigo, Pontevedra; Fig. 1). Fue descubierto en el marco de una obra privada y excavado por la empresa Adro Arqueolóxica, bajo la dirección de María Jesús Iglesias. Junto al yacimiento de igual tipología de la calle O Areal, conforman el sector arqueológico de explotación salinera de época romana encontrado en la ciudad de Vigo. Su localización es en la franja costera, en la parte baja del monte que domina la ciudad, "O Monte Castro". Actualmente está separado del litoral por los rellenos que a partir del último tercio del siglo XIX se sucedieron en paralelo al proceso de crecimiento urbano de la ciudad, para favorecer el ensanche y el acondicionamiento de un puerto comercial (CASTRO CARRERA 2007).

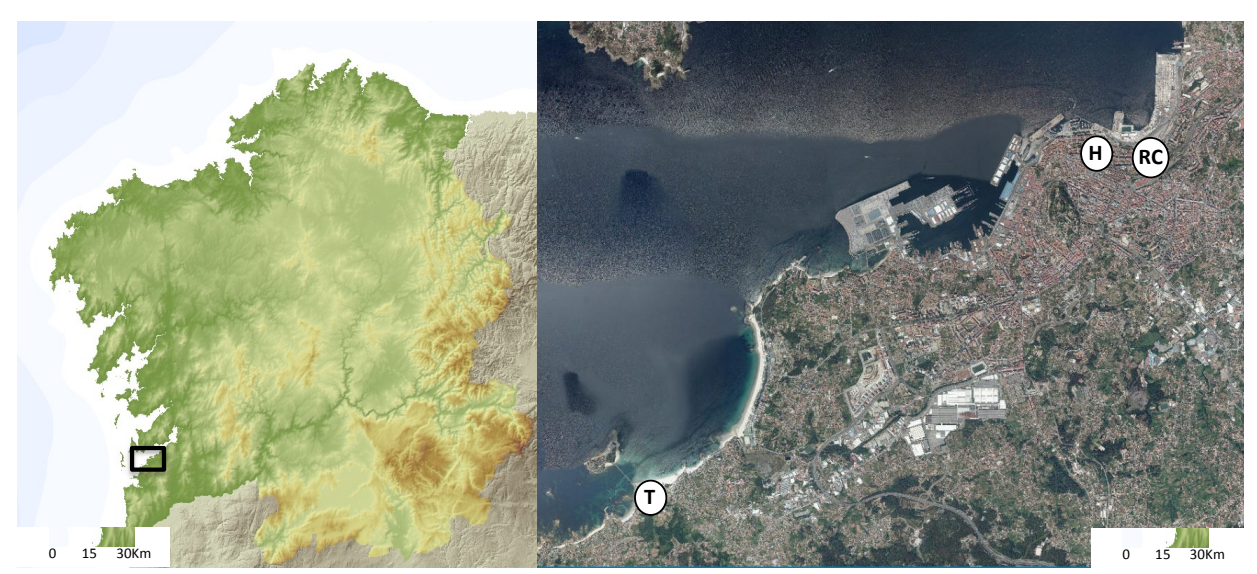

Fig. 1. Mapa de localización de los tres yacimientos comparados a lo largo del artículo. RC: salinas Rosalía de Castro, H: yacimiento de Hospital, T: villa de Toralla. Mapas extraídos de la información mantenida y publicada por la Xunta de Galicia en la web: mapas.xunta.es

Fig. 1. Location map of the three archaeological sites compared throughout this paper. RC: Rosalía de Castro salt mines, H: Hospital settlement, T: villa of Toralla. Maps extracted from the information maintained and published by the Xunta de Galicia on the web: mapas.xunta.es 


\subsection{Metodología}

Se llevó a cabo una descriptiva sedimentológica detallada del área ocupada por el yacimiento de Rosalía de Castro. A partir de ella se identificaron los diferentes ambientes sedimentarios existentes y se seleccionaron tres secuencias estratigráficas por su mayor variabilidad de facies: P3-6-I (potencia vista: $185 \mathrm{~cm}, 47$ muestras), P3-6-II (potencia vista: $90 \mathrm{~cm}, 26$ muestras) y P3-6-III (potencia vista: $75 \mathrm{~cm}, 21$ muestras). En ellas se identificaron 7 niveles estratigráficos (Fig. 2).
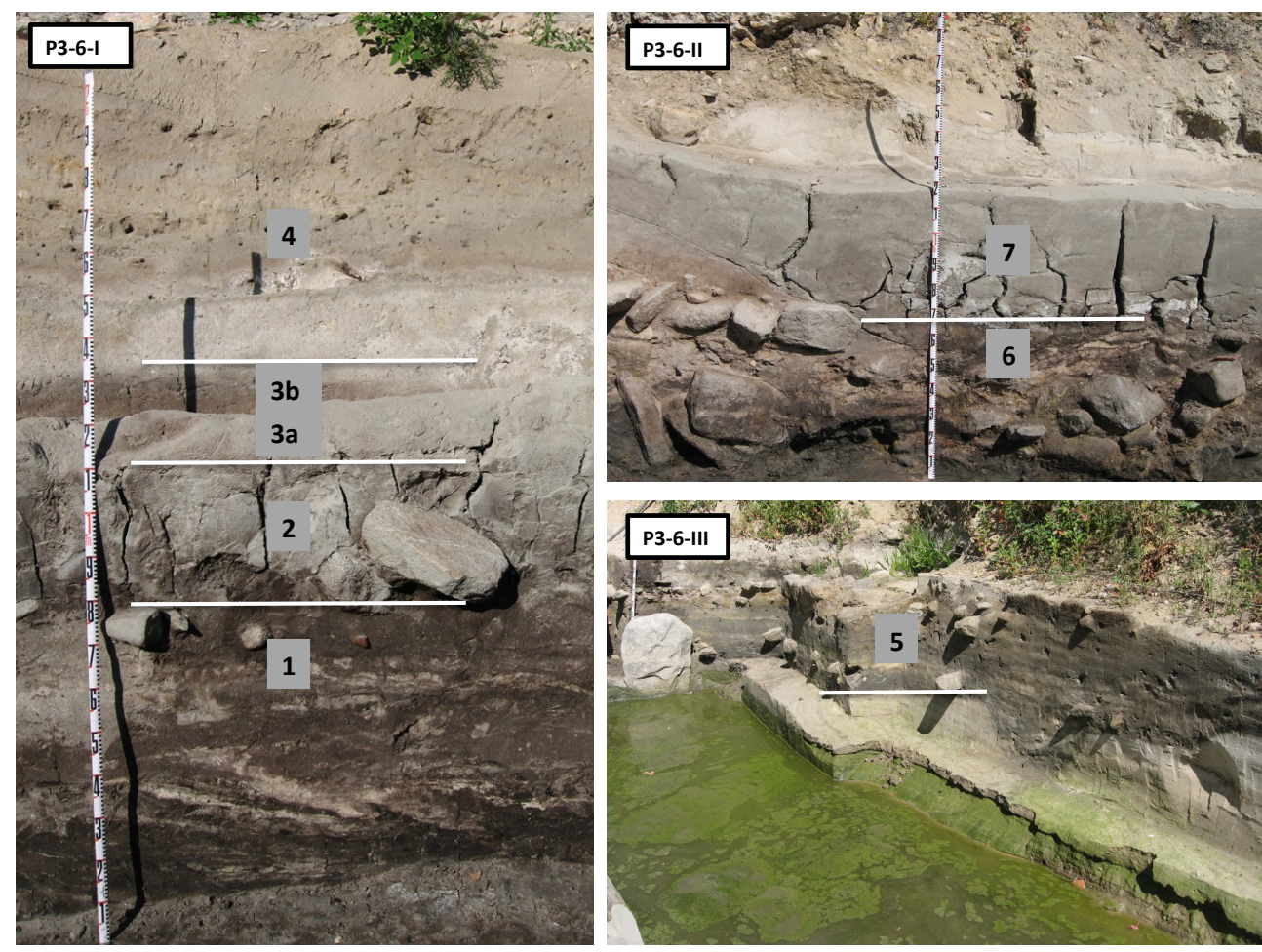

Fig. 2. Estratigrafía vista en campo de las secuencias edafo-sedimentarias seleccionadas en el yacimiento de Rosalía de Castro.

Fig. 2. Field stratigraphy of the pedo-sedimentary sequences of Rosalía de Castro settlement.

La toma de muestras se hizo con alta resolución, tomando secciones continuas de $2 \mathrm{~cm}$ de espesor en los paquetes de sedimentos finos y de 5 $\mathrm{cm}$ en los restantes niveles (a excepción de los niveles con abundancia de clastos). La mayor parte de las analíticas se realizaron sobre la fracción tierra fina $(<2 \mathrm{~mm})$ :

Análisis granulométrico: primero se realizó la desagregación de las partículas, eliminando la fracción coloidal orgánica mediante calcinado a $550{ }^{\circ} \mathrm{C}$ durante 5 horas y posteriormente disolviendo los compuestos de $\mathrm{Fe}$ y $\mathrm{Al}$, añadiendo a cada muestra $32 \mathrm{ml}$ de $\mathrm{HCl} 0,5 \mathrm{~N}$ y agitando durante 20 minutos. Posteriormente se lavaron y filtraron las muestras para eliminar los cloruros. Finalmente se realizó la separación física de cada muestra por cribado con tamices de $0,2 \mathrm{~mm}$ y $0,05 \mathrm{~mm}$, dando como resultado tres fracciones: arena gruesa $(<2 \mathrm{~mm}-0,2$ $\mathrm{mm})$, arena fina $(<0,2 \mathrm{~mm}-0,05 \mathrm{~mm})$ y limo+arcilla $(<0,05 \mathrm{~mm})$.

Reacción del sedimento/suelo ( $\mathrm{pH}$ en auga y $\mathrm{KCl}$ ): el pH se midió en una suspensión suelo/ sedimento $1: 2,5$ en agua (10 minutos de tiempo de contacto; GUITIÁN \& CARBALLAS 1976) y en una disolución de $\mathrm{KCl}$ 0,1 M (tras 2 horas de contacto; URRUTIA et al. 1989).

Las restantes analíticas se realizaron sobre tierra fina molida $(<100 \mu \mathrm{m})$ :

Análisis mineralógico: mediante difracción de rayos X (DRX) en el servicio RIAIDT (Rede de Infraestructura de Apoio á Investigación e ao Desenvolvemento Tecnolóxico, Universidade de Santiago de Compostela), con un equipo Philips con goniómetro PW 1820 .

Análisis de la composición elemental de la materia orgánica $(\mathrm{C}$ y N ; por medio de un analizador LECO, RIAIDT-USC) y de la fracción inorgánica (S, P, Fe, As mediante fluorescencia de rayos $\mathrm{X}$, RIAIDT-USC).

A su vez se enviaron siete muestras para datación radiocarbónica (Beta Analytic Inc., Miami, USA); tres de la secuencia P3-6-I, tres de P3-6-II y una de P3-6-III. Todas las edades mencionadas en este trabajo son fechas radiocarbónicas calibradas, obtenidas con el programa CALIB 6.0.1 (STUIVER \& REIMER 1993). 


\section{RESULTADOS Y DISCUSIÓN}

\subsection{Análisis de facies y cronoestratigrafía}

El estudio conjunto de las tres secuencias permitió obtener un registro edafo-sedimentario completo y detallado, que se caracteriza por presentar alternancia entre niveles arenosos y limoarcillosos, que tienen entre ellos fuertes diferencias en las propiedades físico-químicas (Tabla 1). Esto indica cambios abruptos en los procesos sedimentarios para el periodo de tiempo reflejado por las secuencias estudiadas, ya que los niveles arenosos se encontrarían ligados a un medio más energético, dependiente de la fuerza del viento o el oleaje marino, mientras que la formación de los niveles limo -arcillosos estaría controlada por un medio sedimentario más somero, por ejemplo una marisma, que permitiría la deposición de material fino.

Tabla 1. Relación de dataciones realizadas en las tres secuencias, calibradas y expresadas en BP y BC/AD. Table 1. Calibrated radiorcabon ages of the analysed sequences, expressed in $\mathrm{BP}$ and $\mathrm{BC} / \mathrm{AD}$

\begin{tabular}{|c|c|c|c|c|}
\hline Código Muestra & Profundidad (cm) & Edad ${ }^{14} \mathbf{C}$ BP & $\begin{array}{c}\text { Edad }{ }^{14} \mathbf{C} \text { cal } 2 \text { sig } \\
\text { BP }\end{array}$ & $\begin{array}{c}\text { Edad }{ }^{14} \text { C cal } 2 \text { sig } \\
\text { BC/AD }\end{array}$ \\
\hline P-3-6-IA-05 & & & $1520-1340$ & $420-610 \mathrm{AD}$ \\
\hline P-3-6-IA-22 & 92,5 & $1350 \pm 40$ & $1720-1540$ & $230-410 \mathrm{AD}$ \\
\hline P-3-6-IA-32 & 159,75 & $1720 \pm 40$ & $2100-1880$ & $150 \mathrm{BC}-60 \mathrm{AD}$ \\
\hline & 184,75 & $2020 \pm 40$ & $\mathbf{P - 3 - 6 - I I}$ & \\
\hline P-3-6-II-15 & 36,25 & & $1290-1160$ & $660-780 \mathrm{AD}$ \\
\hline P-3-6-II-22 & 53,75 & $1290 \pm 40$ & $930-750$ & $1020-1200 \mathrm{AD}$ \\
\hline P-3-6-II-26 & 72,5 & $940 \pm 40$ & $1050-920$ & $900-1040 \mathrm{AD}$ \\
\hline
\end{tabular}

La cronología del registro estudiado abarca desde el cambio de era (150-60 BC) hasta el siglo XVII (Tabla X). El estudio de las propiedades y características de los niveles sedimentarios, conjuntamente con la cronología de los sedimentos, permitió hacer una reconstrucción de las distintas facies evolutivas presentes para este medio e inferir los distintos ambientes de formación asociados (Fig.1). Desde la más antigua a la más reciente son:

- Facies I (Fig. 3). Se relaciona con el nivel 1 , que presentan reacción del suelo fuertemente ácida $(\mathrm{pH}<2,5)$, es rico en materia orgánica $(3-8 \%$ carbono), arenas finas ( $>70 \%)$ y restos vegetales; está formado por arenas gruesas y finas con estratificación cruzada. Mineralógicamente es rico en cuarzo $(>40 \%)$, feldespatos $(>20 \%)$, con presencia de fosfatos y alto contenido en azufre (Tabla 2). Estas características junto al hecho de ser el relleno de las estructuras (decantadores) de la salina romana, lo identifican como el nivel de uso de la misma (Figura 3). El alto contenido en fracción fina estaría relacionado con el proceso extractivo de sal, con circulación somera del agua y decantación de materiales en suspensión; la presencia de fosfatos y sulfatos $\mathrm{y}$, el $\mathrm{pH}$ extremadamente ácido ponen de manifiesto la existencia de niveles sulfato-ácidos similares a los descritos en marismas (OTERO \& MACÍAS 2002).

- Facies II. Nivel de cantos que fosilizan el nivel de salina (Fig. 3). Se corresponde con el de- rrumbamiento de las estructuras de la salina.

- Facies III. Se corresponde con el nivel 2 (Tabla 2, Fig. 3). Se caracteriza por un alto contenido en limos y arcillas, materia orgánica (2-13\% de carbono) y azufre y $\mathrm{pH}$ ácido a extremadamente ácido; además presenta mayor contenido en arsénico y fósforo que los restantes niveles. Mineralógicamente está formada por cuarzo ( $>40 \%)$, caolinita (entre 10-37\%), feldespatos potásicos (entre 15$16 \%$ ), plagioclasas (entre 11-17\%) y, en baja abundancia, sulfatos, fosfatos y óxidos de hierro (Tabla 2). Sus características lo adscriben, al igual que el nivel 1, a un suelo de marisma de tipo sulfatoácido (OTERO \& MACÍAS 2002), formado bajo un ambiente de interacción marino-continental, dependiente de la influencia marina y de la acumulación de materia orgánica sedimentaria.

- Facies IV: Esta facies marca la aparición de los niveles arenosos en el sector. Está formada por arenas gruesas y finas, sin estructura, enriquecidas en cuarzo $(>50 \%)$, con características morfológicas eólicas (granos con marcas de punteado, superficies mates) y pH neutro, nivel 3a (Tabla 2, Fig. 3). Este nivel se asocia a una facies tipo duna, su morfometría y mineralogía lo caracterizan como eólico y su origen estaría relacionado con una mayor o menor intensidad del viento. El material que lo conforma, arenas gruesas ricas en carbones, se hace más fino hacia el techo de la unidad a la par que presentan una estructura edáfica; las muestras 


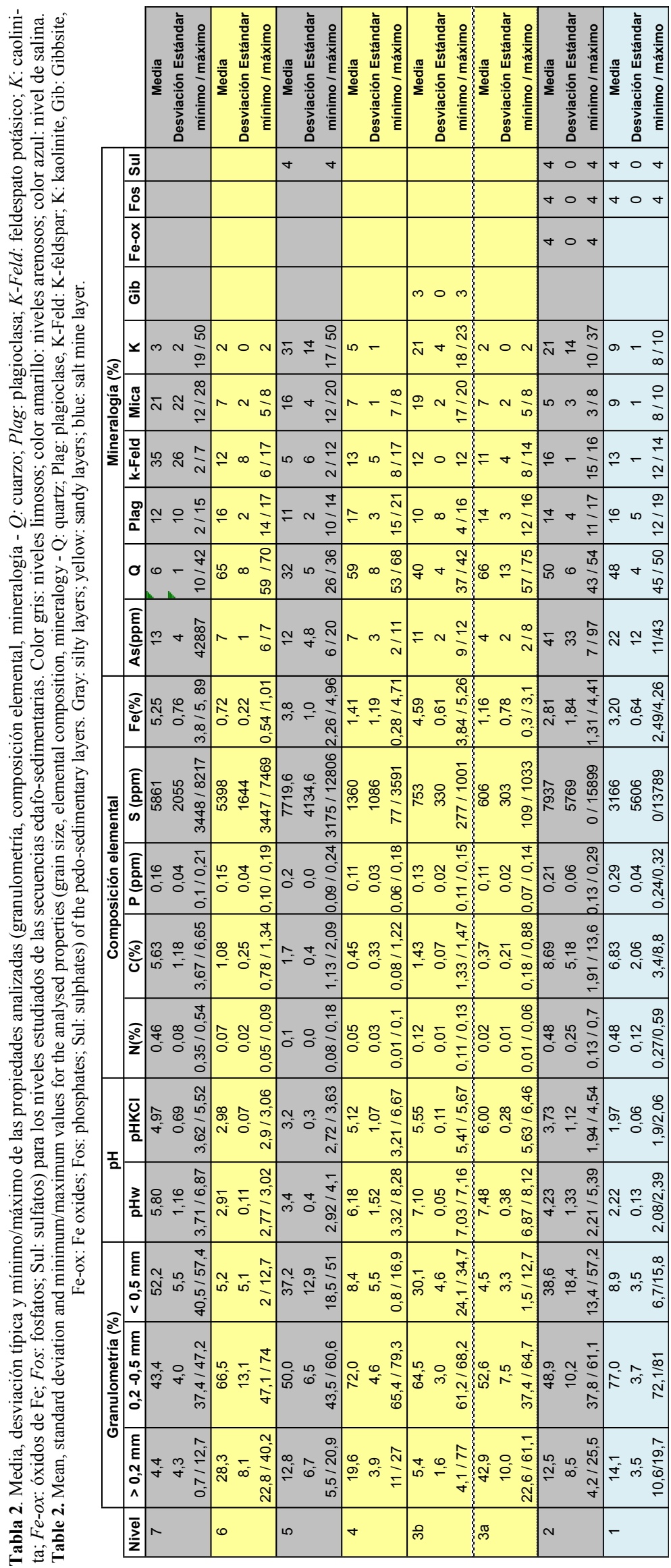


superiores presenta menor contenido en cuarzo, enriquecimiento en Fe, caolinita (18-23\%, Tabla 2) y micas (17-20\%), pH ligeramente ácido y moderado contenido en materia orgánica $(1-1,5 \%$ carbono). Estas propiedades son rasgos de edafogénesis que sugieren que se trata de un horizonte de suelo enterrado (un epipedon, nivel 3b, Tabla 2, Fig. 3). $\mathrm{Su}$ formación necesariamente implicó un periodo de cierta estabilidad de la duna, que permitiría la aparición de una cobertura vegetal y el desarrollo de un suelo sobre sedimentos arenosos. Este nivel tiene las características de una duna parda (MARTíNEZ et al. 1996); aquellas que se forman en la zona más alejada del mar, con mayor estabilidad y con un grado de evolución edafogenético medioalto. A su vez, la facies IV representa una ruptura en cuanto a las formaciones anteriores del área, que son de origen marino, o marino-continental, frente a los sistemas dunares que implican una mayor influencia eólica.

- Facies V: En esta facies continúan dominando las formaciones dunares, nivel 4 , con características similares al nivel $\mathbf{3 b}$, aunque con una granulometría más fina. Tras el periodo de estabilidad y edafogénesis representado por el nivel anterior, los procesos eólicos parecen haberse reactivado para dar lugar a una segunda fase dunar, formada principalmente por sedimentos de arenas finas sin estructura (Tabla 2, Fig. 3). Se trata de una duna blanca (MARTíNEZ et al. 1996) desprovista de vegetación y sin signos de edafogénesis o estabilización.
Mientras se desarrollan las formaciones dunares, reaparecen niveles limo-arcillosos y arenosos formados bajo distinto grado de interacción marino continental. Los niveles más ricos en finos presentan moderado contenido en materia orgánica (1-6\% carbono) y alto contenido en caolinita $(>25 \%)$ y micas $(>15 \%)$. Dentro de ellos, los que corresponden al nivel 5 tienen además alto contenido en azufre, trazas de sulfatos y $\mathrm{pH}$ extremadamente ácido a ácido $(\mathrm{pH}$ entre 2,9-4,1, características sulfatoácidas), mientras que los del nivel 7 presentan un bajo-moderado contenido en azufre y $\mathrm{pH}$ ácido a ligeramente ácido $(\mathrm{pH}$ entre 3,7-6,9) (Tabla 2).

Los niveles arenosos son extremadamente ácidos ( $\mathrm{pH}$ 2,7-3,0), ricos en azufre, con bajo contenido en materia orgánica $(0,7-1 \%$ de carbono); están dominados por arena fina $(>40 \%)$, y enriquecidos en cuarzo ( $>50 \%$ ) (Tabla 2, Fig. 3, nivel 6).

Las características de estos niveles se pueden relacionar con formaciones de marisma (niveles 5 y 6) o canales de marisma/lagoon costero (nivel 7), dependiendo de la interacción entre los procesos marinos y los continentales (oleaje marino y cursos de agua dulce) durante su formación.

La coexistencia de estas formaciones indica que aunque los procesos eólicos son los que dominan la dinámica del sector, no lo hacen de forma exclusiva, ya que para momentos puntuales se produce un retorno de formaciones de marisma/ canales de marisma/lagoon costero. Esto se debe a la variación espacial de los procesos sedimentarios a pequeña escala, así como, al dinamismo de los sistemas litorales y su sensibilidad a los cambios.

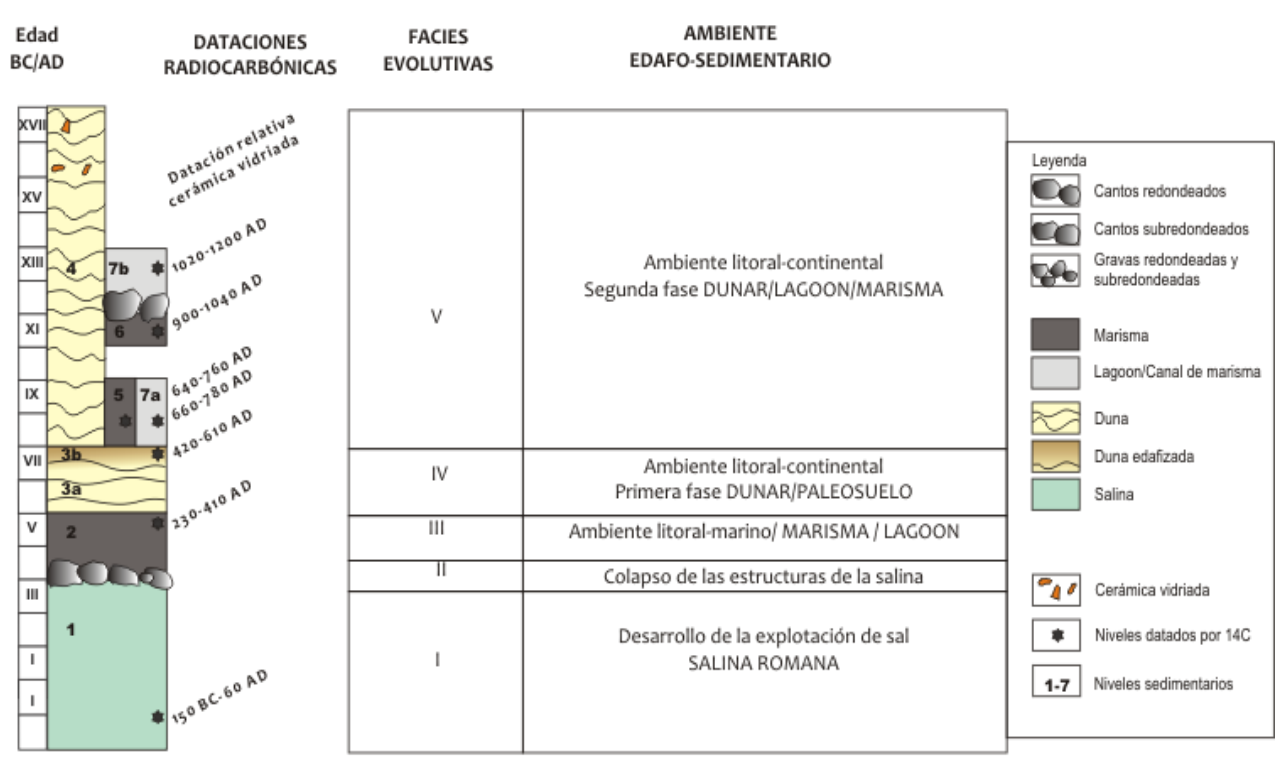

Fig. 3. Cronestratigrafía del registro estudiado e interpretación de los ambientes de formación.

Fig. 3. Cronoestratigraphy of the studied sequences and interpretation of the formation environments. 


\subsection{Episodios ambientales y culturales en la Alta Edad Media}

En función de las dataciones obtenidas se pueden correlacionar las facies evolutivas con distintos periodos culturales y climáticos (Fig. 4).

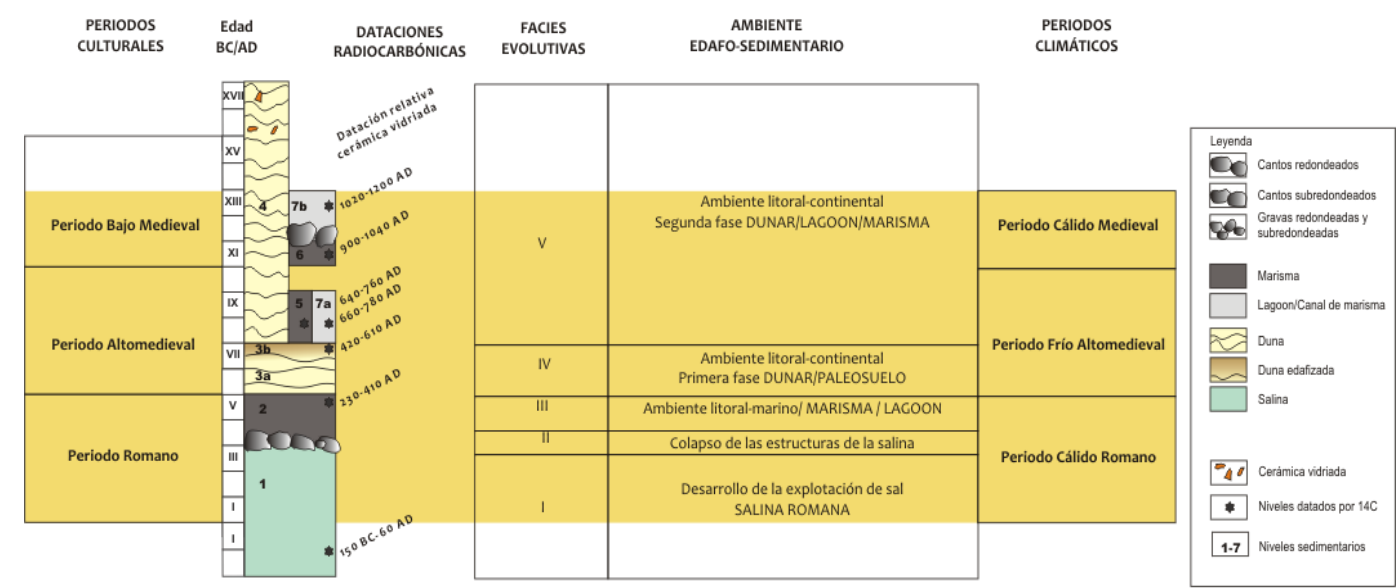

Fig. 4. Cronoestratigrafía y relación con periodos culturales y episodios climáticos para el intervalo Periodo RomanoPeriodo Bajo Medieval.

Fig. 4. Chronostratigraphy, cultural and climate phases for the interval from the Roman Period to the late Middle Ages.

\section{Periodo Romano (S. II/III-S. IV-V AD)}

Este periodo se caracteriza por la explotación de sal iniciada en torno a los siglos II/I BC, con cese de la actividad y derrumbamiento de las estructuras en torno a los siglos II/III AD (Facies I y II, Fig. 4). Este periodo finaliza con una facies de marisma que colmata las estructuras de la salina. La fase climática en la cual se adscribe es el Periodo Cálido Romano (100 BC-400 AD) que supone la etapa final del calentamiento irregular y de la fase transgresiva marina que comienza en torno al 1000 BC (MARTínez CorTizas et al. 1999). Según los datos disponibles para el noroeste peninsular, se caracteriza por una mejora climática con una temperatura unos $2,5-3^{\circ} \mathrm{C}$ superior y una precipitación hasta un 75\% mayor que la actual (MARTíNEZ CORTIZAS \& VÁZQUEZ VARELA 2002); si bien hay que diferenciar entre una fase inicial (hasta el siglo II $\mathrm{AD}$ ) y una fase final (a partir del siglo II AD). La fase inicial se caracteriza por escasas precipitaciones (del orden de la mitad a las tres cuartas partes de la que se recoge actualmente) y temperaturas similares a las actuales mientras que a partir del siglo III AD es cuando se produce un aumento de la temperatura y de la precipitación (MARTíNEZ CORTIZAS \& VÁZQUEZ VARELA 2002).

Tanto el clima cálido y seco como una estabilización o ligera subida del nivel del mar durante la fase inicial podrían explicar el desarrollo de la salina a una latitud tan alta de la costa atlántica peninsular, un enclave en principio no preparado ni climáticamente, ni topográficamente para ello (ALONSO VillalobOS \& MÉNANTEAU 2006; CURRÁs 2007).

El cese de la actividad salinera ocurre en torno al siglo III AD, momento en el que también cesan las factorías de salazón identificadas en la Ría de Vigo (CURRÁs 2007) y en sectores de las Rías Altas (PIÑEIRO 2003). Entre los siglos III-V $\mathrm{AD}$ se detecta un aumento de la precipitación tanto para el NW peninsular (MARTÍNEZ CORTIZAS \& VÁZQUEZ VARELA 2002) como para Centroeuropa (BÜNTGEN et al. 2011), a la par que otros autores identifican un deterioro climático entre el 270-385 cal AD (SCHELlEKENS et al. 2010; CRUCES et al. 2011). Esta fase más húmeda pudo implicar peores condiciones para la explotación de sal con el acortamiento del periodo útil de evaporación.

\section{Alta Edad Media ( $V-X A D)$}

En este periodo se produce un importante cambio en los procesos sedimentarios, coincidiendo con el final del Periodo Romano y comienzo de la Alta Edad Media. El medio evoluciona desde la formación de marismas, hacia un ambiente eólico con dunas que predominará hasta el siglo XVII AD (datación relativa de la parte superior del registro, Fig. 4). El material eólico sella los niveles de salina y marisma y marca el comienzo de una nueva fase de predominio dunar.

Este cambio es coincidente climáticamente con el inicio del Periodo Frío Altomedieval (siglos $\mathrm{V}$-IX AD), que presenta características inversas al anterior, con bruscas variaciones térmicas a comienzos del siglo $\mathrm{V}$ y un régimen de precipitación irregular seguido de etapas de intenso frío y sequía durante los siglos VI, VII, y VIII AD (FONT TULLOT 1988). Consecuentemente la dinámica litoral 
cambia produciéndose una intensificación de la actividad eólica que removilizó y depositó estos materiales arenosos.

Entorno a los siglos VI/VII AD, se forma un paleosuelo sobre duna (duna parda, MARTínEZ CORTIZAS et al. , 1996), al mismo tiempo que reaparecen los niveles marisma/canales de marisma/ lagoon costero (niveles 5 y 7a, Tabla 2, Figura 4). La edafogénesis sobre duna implica estabilidad superficial y colonización biológica, mientras que la acumulación de limos implica circulación de agua somera. Ambas características estarían asociadas a condiciones más cálidas que las descritas para un episodio frío. Mientras el periodo de degradación climática persiste en el resto de Europa durante todo el Episodio Frío Altomedieval, en Galicia, entre el 650 y el 1000 cal BP, las condiciones son más benignas, secas y relativamente cálidas, semejantes a las del periodo cálido romano (COSTA-CASAis et al. en esta publicación). Esta mejoría también se observa en Centroeuropa a partir del siglo VI (BÜNTGEN et al. 2011). Unas condiciones más benignas facilitarían le estabilidad superficial y la edafogénesis, así como la reactivación de las marismas asociadas a una mayor circulación de agua, tanto salada como dulce. A pesar de ello, no hay indicios de una vuelta a la explotación de sal en el sector, aunque sí se produce un cambio en el uso antrópico, con la implantación de una necrópolis y una iglesia, que ocuparon el espacio de las salinas a finales del siglo VI y primer tercio del siglo VII (MEMORIA DE EXCAVACIÓN 2007).

Tras este momento de condiciones más benignas, se produce una reactivación de los aparatos dunares y el comienzo de una nueva fase de formación de dunas (duna blanca, MARTÍNEZ CORTIZAS et al. 1996) que se mantendrá hasta siglo XVII AD, por lo menos (Facies V, Fig. 1). Dicha evolución implica cambios en los aparatos sedimentarios que conllevaron el paso de una zona dunar estable, colonizada por la vegetación y relativamente alejada del mar (representada por el paleosuelo), a una zona activa, cercana a la línea de costa. Al mismo tiempo que comienza la formación de una nueva fase dunar, continúa la formación de los niveles limosos (niveles 5 y 7a, facies V, Fig. 4).

\section{Baja Edad Media (X-XVII AD)}

En este periodo el avance dunar prosigue a la par que las formaciones de marisma (niveles $6 \mathrm{y}$ $7 b$, Facies V, Fig. 4). La primera parte de esta fase se correlaciona con el Periodo Cálido Medieval (900/1000-1300 AD), en el que las temperaturas pudieron ser hasta $3,5{ }^{\circ} \mathrm{C}$ superiores a las actuales (MARTíneZ CORTIZAS et al. 1999). En esta fase las condiciones se hacen paulatinamente más suaves y más húmedas en la vertiente atlántica de la Península Ibérica en comparación con la vertiente mediterránea, donde se tornan más áridas (MORENO et al. 2012). Para Centroeuropa esta etapa se caracteriza por una disminución de la variabilidad climática, dominando los veranos cálidos y húmedos y temperaturas similares a las del periodo romano (BÜNTGEN et al. 2011). Cabe destacar que al final de este periodo se detecta un evento erosivo. Esto queda reflejado en la edad del nivel 7 (Fig. 4). La parte basal del nivel 7 , tiene una edad más reciente (7b) que la parte superior (7a) (Tabla 1, Fig. 4), lo que indica que en algún momento durante su formación se produjo la exhumación de formaciones más antiguas y erosión en el entorno, depositándose materiales limo-arcillosos más antiguos sobre el nivel actual en aquel momento.

\subsection{Uso y evolución del sector de Rosalía de Castro. Comparativa con los sectores de Toralla y Hospital}

Uno de los principales usos antrópicos del sector estudiado fue la explotación de sal. Dicha actividad, que pareció tener alta relevancia dentro del sistema industrial romano del noroeste (CURRÁs 2007), no se vuelve a retomar durante la Alta Edad Media, ni tampoco en épocas posteriores en este área, desde su cese en torno al siglo III AD. Un clima más cálido y seco durante el Periodo Romano facilitaría la implantación de la explotación de sal marina. Mientras que el aumento de la precipitación al final de Periodo Romano (formación de marismas) y el posterior avance dunar durante la Alta Edad Media (asociado al Periodo Frío Altomedieval), limitarían la obtención de sal por evaporación. Tal y como apuntan estudios recientes, los cambios sociales de los últimos 2500 años se correlacionan con las distintas variaciones climáticas, entre ellas la transición entre los periodos culturales romano y medieval (BÜNTGEN et al. 2011). Esto implica que el clima podría haber desempeñado un papel importante en la evolución y uso del sector entre el Periodo Romano y la Alta Edad Media. Aún así, los cambios en la costa pudieron ocurrir tanto por causas climáticas como socioeconómicas, o una combinación de ambas. Una vez finalizada la explotación de sal no se vuelven a encontrar indicios de actividad antrópica en el sector hasta el siglo VI AD, tal y como hemos mencionado anteriormente.

Los registros costeros de los yacimientos galaico-romanos de Toralla y Hospital (MARTínEZ CORTIZAS \& COSTA CASAIS 1997) presentan un patrón de evolución litoral muy semejante al de Rosalía de Castro. En el primero de ellos, Toralla (Fig. 5), el medio evoluciona desde un sistema de marisma hacia un ambiente dominado por procesos eólicos. En este caso, la formación de los niveles dunares aparece con cronología anterior a Rosalía de Castro, uno en torno al III AD y otro posterior al VI AD, según los datos arqueológicos, por lo que los autores proponen un punto de cambio ambiental asociado al final del Periodo Cálido Romano. Por otro lado, en Hospital (Fig. 5), a partir del 


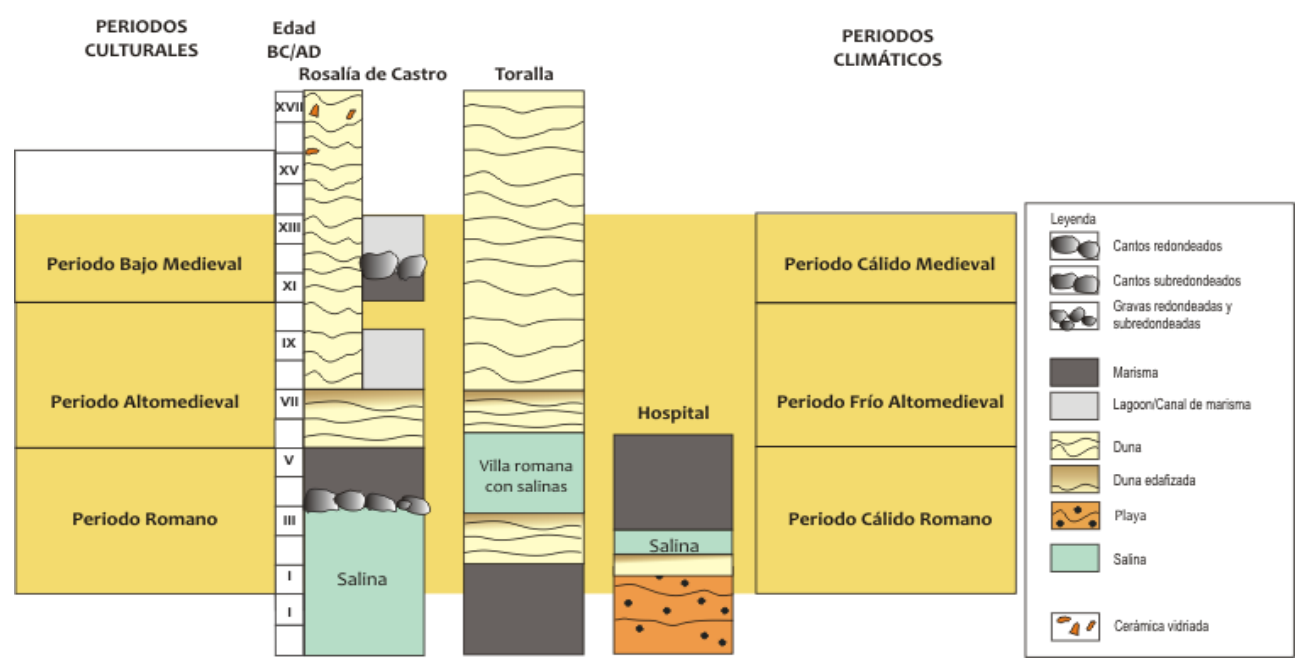

Fig. 5. Cronoestratigrafía, períodos culturales y fases climáticas para las secuencias de Rosalía de Castro, Toralla y Hospital (para las dos últimas la información se extrae de MARTínez CORTIZAS \& COSTA CASAIS 1997).

Fig. 5. Chronostratigraphy, cultural periods and climate phases of Rosalia de Castro, Toralla and Hospital sequences (the last two after MARTINEZ CORTIZAS \& COSTA CASAIS 1997).

cambio de Era es cuando se produce la entrada de material fino continental que fosiliza un nivel basal de arenas marinas y culmina con la formación de una marisma en torno al siglo III AD. En ambos sectores aparecen restos arqueológicos asociados a la explotación de sal. En Toralla se relacionan con una villa romana cuyo funcionamiento se enmarcaría en torno a los siglos IV-V AD (PEREZ LOSADA et al. 2005, 2008; MARTíNEZ CORTIZAS \& COSTA CASAIS 1997), por lo que cronológicamente serían posteriores a los encontrados en Rosalía de Castro. En Hospital aparecen unas estructuras de salina con cronología en torno al siglo I-II AD, coetáneas a Rosalía de Castro, aunque su explotación parece haber tenido una menor duración.

En los tres sectores el principal cambio de uso y de la sedimentación costera ocurrió entre final del periodo romano y comienzos de la Alta Edad Media (Fig. 5). En este momento se produjo el avance de los aparatos eólicos (progradación dunar) junto a un empeoramiento climático (Periodo Frío Altomedieval). Estas modificaciones de la línea de costa son coincidentes con el cese de la explotación de sal, actividad que no llega más allá de los siglos III-VI AD en ninguno de los sectores, a pesar de la mejoría climática que se detecta para Galicia en torno a los siglos VI-VII AD (COSTA CASAIS, esta publicación).

La salazón en Galicia a nivel industrial tiene un amplio desarrollo en época romana, principalmente en las Rías Baixas. Aparecen distintas zonas con salinas, factorías de salazón y conserva de pescado sobre los siglos I-II AD, cuyo funcionamiento se extendería aproximadamente hasta el siglo III AD y se encontrarían organizadas en relación al puerto comercial de O Monte do Castro de Vigo (CURRÁs 2007). Los sectores de Rosalía de Castro, Toralla y Hospital, probablemente formar- ían parte de esta estructura industrial, cuyo funcionamiento no llegaría más allá del siglo III-V, siendo Toralla el sector con una cronología más tardía (hasta los siglos IV-V AD) (PEREZ LOSADA et al. 2005, 2008). En el sector de Toralla el avance dunar parece ser una causa importante en el abandono de la villae y de la explotación de sal; mientras que en los otros dos casos, de cronología más temprana, tras el abandono de la actividad salinera el medio se reorganiza, apareciendo las marismas antes del desarrollo de las formaciones dunares. Es por ello que no se puede afirmar que la causa determinante de los cambios en la línea de costa sea exclusivamente de tipo climático, sino más bien de tipo climático y/o antrópico. En general, hasta época Bajomedieval, la explotación de sal no recobra auge en las costa de Galicia (CASTIÑEIRA CASTRO 1999; CALO LOURIDO 1997).

\section{CONCLUSIONES}

El estudio de las secuencias edafo-sedimentarias en un contexto de explotación de sal de época romana (Calle Rosalía de Castro, Vigo, NW España), muestra una evolución del medio marcada por la progradación dunar, que se intensifica a partir del inicio de la Alta Edad Media, asociada a un periodo climático frío y seco (Periodo Frío Altomedieval). Dicho periodo presenta, para el NW de la Península Ibérica, una pulsación cálida entre los siglos VI-VII AD, posiblemente atestiguada por la estabilización y edafización de las dunas (formación de un paleosuelo) durante este periodo.

La explotación de sal desarrollada en época romana, cesa en torno a los siglos III-V (al igual que el resto de industria de salazón) y no es retomada durante la Alta Edad Media, ni en periodos posteriores, en el área de estudio. Se observa una secuencia 
de cambios en la línea de costa que es coincidente con las distintas variaciones climáticas detectadas para el NW peninsular. Un clima más cálido y menor precipitación durante la primera mitad del periodo romano pudo facilitar el desarrollo de la actividad salinera. El aumento de la precipitación al final de Periodo Romano y un clima más frío durante la Alta Edad Media habrían determinado una reestructuración de los aparatos sedimentarios que culminó en una progradación dunar, pudiendo estas condiciones dificultar la explotación de sal u otras actividades desarrolladas en el litoral. Aunque la pauta climática presenta un papel importante en la evolución del área de estudio, los cambios reconstruidos pudieron estar influidos tanto por causas climáticas como socioecómicas, o una combinación de ambas.

El proceso de progradación dunar identificado en Rosalía de Castro también se observa en otros lugares cercanos, dentro de la Ría de Vigo (yacimientos arqueológicos de Toralla y Hospital), con un rango cronológico similar para el desarrollo y abandono de la actividad salinera. En la Alta Edad Media no hubo explotación de sal en el área, aunque sí aparecen otras estructuras antrópicas resultado de otros usos del sector, como una iglesia y su necrópolis asociada.

\section{Agradecimientos}

Parte de esta investigación fue realizada en el marco del contrato/convenio "Toma de mostras e estudio paleoambiental no xacemento arqueóloxico de Rosalía de Castro" 2007-CP267, entre la Universidad de Santiago de Compostela y la Dirección Xeral de Patrimonio Cultural-Xunta de Galicia y Rede Consilencia R2014/001 y GPC2014/009 financiados por la Xunta de Galicia.

\section{BIBLIOGRAFÍA}

Abrantes, F.; Lebreiro, S.; Rodrigues, T.; Gil, I.; BARTELS-JÓNSDÓtTIR, H.; OlIVEIRA, P.; KisSEl, C. \& GRIMALT, J.O. 2005. Shallow-marine sediment cores record climate variability and earthquake activity off Lisbon (Portugal) for the last 2000 years. Quaternary Science Reviews, 24:2477-2494.

Alonso, A. \& PAGÉs, J.L. 2007. Stratigraphy of Late Pleistocene coastal deposits in Northern Spain. Journal of Iberian Geology, 33:207-220.

Alonso Villalobos, C. \& MÉnanteau, L. 2006. Paléoenvironnements et techniques de production du sel marin (par ignition ou insolation) durant l'Antiquité: les cas des baies de Bourgneuf (France) et de Cadix (Espagne), In: Hocquet, J.-C., Sarrazin, J.-L. (Eds.), Le Sel de La Baie. Historie, Archéologie, Ethnologie Des Sels Atlantigues. Collection Historie. Nantes:87-103.

Andrade, A.; Rubio, B.; Rey, D.; Álvarez-Iglesias, P.; Bernabeu, A.M. \& FedI, M. 2014. Environmental changes at the inner sector of Ría de Muros (NW Spain) during Middle to Late Holocene. Estuarine, Coastal and Shelf Science, 136:91-101.
ARAúJo, M. 2008. Depósitos do Pleistocénico superior e do Holocénico na plataforma litoral da região do Porto: a morfologia das plataformas de erosão marinha e a tectónica recente. Estudos do Quaternário, 5:17-30.

Bao, R.; Alonso, A.; Delgado, C. \& Pagés, J.L. 2007. Identification of the main driving mechanisms in the evolution of a small coastal wetland (Traba, Galicia, NW Spain) since its origin $5700 \mathrm{cal}$ yr BP. Palaeogeography, Palaeoclimatology. Palaeoecology, 247:296-312.

Baptista, P.; Coelho, C.; Pereira, C.; Bernardes, C. \& Veloso-Gomes, F. 2014. Beach morphology and shoreline evolution: Monitoring and modelling medium-term responses (Portuguese NW coast study site). Coastal Engineering, 84:23-37.

Blanco-Chao, R.; Pérez-Alberti, A.; Trenhaile, A. S.; Costa-CAsAis, M. \& VALCÁRCEL-DíAz, M. 2007. Shore platform abrasion in a para-periglacial environment, Galicia, northwestern Spain. Geomorphology, 83:136-151.

BÜntgen, U.; Tegel, W.; Nicolussi, K.; MCCormick, M.; Frank, D.; Trouet, V.; Kaplan, J.O.; Herzig, F.; HEUSSNER, K.-U.; WANNER, H.; LUTERBACHER, J. \& ESPER, J. 2011. 2500 years of European climate variability and human susceptibility. Science, 331:578-82.

CAlo Lourido, F. 1997. Notas para o estudio do sal en Galicia. Peripecias dunhas salinas. Portugalia, XVII-XVIII:211-217.

CARTER, R.W.G. 1988. Coastal environments. An introduction to the physical, ecological and cultural systems of coastlines. Londres. Academic Press. 617 pp.

CARTER, R.W.G. \& WoOdRoffe, C.D. 1994. "Coastal evolution: an introduction". EN R.W.G. CARTER AND C.D. WoOdroffe (eds.) Coastal evolution. Late Quaternary shoreline morphodynamics. Cambridge: $1-31$

Carvalhido, R.P.; Pereira, D.I.; Cunha, P.P.; Buylaert, J.-P. \& MurRAY, A.S. 2014. Characterization and dating of coastal deposits of NW Portugal (MinhoNeiva area): A record of climate, eustasy and crustal uplift during the Quaternary. Quaternary International, 328-329:94-106.

CASTIÑEIRA CASTRO, V.M. 1999. El litoral gallego y el abastecimiento del sal a mediados del S.XVI. Obradoiro de Historia Moderna, 8:7-30.

CASTRO CARRERA, J. C. 2007. La salina romana del yacimiento de "O Areal" Vigo (Galicia): un complejo industrial salazonero altoimperial. En: Langóstena, L. , Bernal, D., Arévalo, A. (eds.), Salsas y salazones de pescado en Occidente durante la Antigüedad, Actas del Congreso Internacional CETARIAE, 355-365. British Archaeologiacl Reports, International Series 1686.

Costa-CASAIS, M. 2001. Análise sedimentaria e reconstrucción paleoambiental da costa Atlántica de Galicia. Servicio de Publicacións e Intercambio Científico. Universidade de Santiago de Compostela, $236 \mathrm{pp}$.

Costa-Casais, M.; Blanco Chao, R.; Martínez Cortizas, A. \& Pérez Alberti, A. 2007-2008. Los sedimentos Heinrich en la costa de Galicia (NW de la Península Ibérica). Un análisis a través de los sedimentos continentales. Revista Territoris, 7:39-53. 
Cruces, A.; Quaresma, J. C.; Freitas, M.C.; Andrade, C.; FERREIRA, T. \& ARAÚJO, F.M.. 2011: Evidências geológicas e arqueológicas para a transição climática entre o Período quente romano e o "Período das trevas" no SW alejentano. En: Almeida, A.C.; Bettencourt, A.M.S.; Monteiro Rodrigues, S.; Caetano Alves M.I.; Moura, D. (eds); Abstracts book: "Alteraçoes ambientais e interação humana na fachada atlântica ocidental". IV Jornadas do Quaternário, 1st International Conference, Coimbra.

CURRÁs, B. 2007. Aportacion al conocimiento de la industria de salazon en las Rias Baixas gallegas. En: Langóstena, L., Bernal, D., Arévalo, A. (Eds.), "Salsas y salazones de pescado en Occidente durante la Antigüedad", Actas Del Congreso Internacional CETARIAE. Oxford, British Archaeological Reports, International Series 1686:135-149.

De Carvalho, G.S.; Granja, H.M.; Loureiro, E. \& HenRIQUES, R. 2006. Late Pleistocene and Holocene environmental changes in the coastal zone of northwestern Portugal. Journal of Quaternary Science, 21:859-877.

Dias, J.M.; GonZalez, R., Garcia, C. \& DiaZ-DEL-Rio, V. 2002. Sediment distribution patterns on the GaliciaMinho continental shelf. Progress in Oceanography, 52:215-231

FÁBregas VAlCARCE, R.; Martínez Cortizas, A.; Blanco ChaO, R. \& Chesworth, W. 2003. Environmental change and social dynamics in the second-third millennium BC in NW Iberia. Journal of Archaeological Science, 30:859-871.

FONT Tullot, I.; 1988. Historia del clima en España. Cambios climáticos y sus causas. Ministerio de Transportes, Turismo y Comunicaciones. Instituto Nacional de Meteorología. Madrid. 297 pp.

Gomez-Gesteira, M.; Beiras, R.; Presa, P. \& Vilas, F. 2011. Coastal processes in northwestern Iberia Spain. Continental Shelf Research, 31:367-375.

Granja, H.; Rocha, F.; Matias, M.; Moura, R.; CALdas, F.; Marques, J. \& TARECO, H. 2010. Lagoa da Apúlia: A residual lagoon from the Late Holocene (NW coastal zone of Portugal). Quaternary International, 221:46-57.

Granja, H.M.; Dte Groot, T. A. M. \& Costa, A.L. 2008 Evidence for Pleistocene wet aeolian dune and interdune accumulation, S. Pedro da Maceda, northwest Portugal. Sedimentology, 55:1203-1226.

Granja, H.M.; Ribeiro, I.C. \& Matias, M.S. 1999. Some Neotectonic Indicators in Quarternary Northwest Coastal Zone of Portugal. Physics and Chemistry of the Earth, Part A, 24:323-336.

Guitián \& Carballas 1976. Técnicas de Análisis de suelos. Santiago de Compostela, Pico Sacro.

Martínez Cortizas, A.; Costa Casais, M. \& MoAres DOMÍNQUEZ, C. 1996. Niveles dunares pleistocenos y holocenos en la costa de Galicia: hipótesis cronológica en base a su grado de edafización. En: Pérez Alberti, A., Chesworth, W., Martínez Cortizas, A. (eds.), Dinámica y Evolución de Medios Cuaternarios, Xunta de Galicia, 417-430.

Martínez Cortizas, A. \& Costa Casais, M. 1997. Indicios de variaciones del nivel del mar en la Ría de Vigo durante los últimos 3000 años. Gallaecia, 16:23-47.
Martínez Cortizas, A.; Castillo Rodríguez, F.; PÉrez Alberti, A.; VAlCÁrcel DíAZ, M. \& Blanco CHAO, R. 1999. Atlas Climático de Galicia. Santiago de Compostela, Xunta de Galicia.

Martínez Cortizas, A. \& VÁzquez Varela, J.M. 2002. El clima en la Galicia romana: una aproximación interdisciplinar. Revista Real Academia Galega das Ciencias, XXI:87-104.

MartíneZ-CortizAS, A.; COSTA-CASAIS, M. \& LÓPEZ SÁEZ, J.A. 2009. Environmental change in NW Iberia between 7000 and 500 cal BC. Quaternary International, 200:77-89.

MeIRELES, J. \& TeXIER, J.P. 2000. Etude morphostratigraphique des dépôts littoraux du Minho (NW du Portugal). Quaternaire, 11:21-29.

MEMORIA DE EXCAVACIÓN 2007. Fase II evaluación patrimonial de la unidad de actuación I-06. Rosalía de Castro II, Vigo (Pontevedra). Clave; CD 102 A 2006/368-0

Moreno, A.; PÉrez, A.; Frigola, J.; Nieto-Moreno, V.; Rodrigo-GÁMIZ, M.; MARTRAT, B.; GONZÁleZSAMPÉRIZ, P.; MOREllón, M.; MARTÍN-PUERTAS, C.; Corella, J.P.; Belmonte, Á.; Sancho, C.; Cacho, I.; Herrera, G.; Canals, M.; Grimalt, J.O.; JIMÉNEZ-ESPEJO, F.; MARTÍNEZ-RUIZ, F.; Vegas-VilarRúbia, T. \& VALERO-GARCÉS, B.L. 2012. The Medieval Climate Anomaly in the Iberian Peninsula reconstructed from marine and lake records. Quaternary Science Reviews, 43:16-32.

Otero, X.L. \& Macías, F. 2002. Fracionamiento de Fe en fluvisoles de las marismas de la Ría de Ortigueira (Galicia). Edafología, 9:257-272.

Pérez Losada, F.; Vieto Covela, S. \& FernándeZ FERNÁNDEZ, A. 2005. La villa romana de T (Vigo): una innovadora propuesta de tratamiento y presentacióin de un yacimiento arqueológico excavado. En: Francia Gómez, C., Erice Lacabe, R. (coords.), Congreso Internacional sobre Musealizacion de yacimientos arqueológicos. De la excavación al público: procesos de decisión y creación de nuevos recursos. 2004. Institución Fernando Católico. Ayuntamiento de Zaragoza.116-122.

Pérez Losada, F; Fernández Fernández , A \& Vieto COVELA, S. 2008.Toralla y las villas maríticas de la Gallaecia atlántica. Emplazamiento, arquitectura y función. En: Fernández Ochoa, C., García-Entero V. \& Gil Sendino, F. (eds.), Las villae tardorromanas en el occidente del Imperio: arquitectura y función. IV Coloquio Internacional de Arqueología en Gijón. 481-506.

PIÑEIRO, A.M.S. 2003. La explotación del mar en la Galicia romana: el ejemplo de las instalaciones de salazón. Cuadernos de Estudios Gallegos, 116:9-25.

TALlón ARMADA, R. 2012. Marco evolutivo dunha explotación salineira de época romana (Vigo, Pontevedra). Memoria de Licenciatura. Universidade de Santiago de Compostela.

TAllón Armada, R.; Costa-Casais, M.; TABoAda, T. \& MARTÍNEZ CORTIZAS, A. 2013. Reconfiguración de la línea de costa en un sector de explotación salinera de época romana en el NO de España. En: Flor Rodríguez, G., Flor-Blanco, G, Pando Gonzalez, L.A.(eds.), Geotemas, VII Jornadas de Geomorfología Litoral, Volumen 14, Sociedad Geológica de España. Oviedo. 211-214. 
Schellekens, J.; BuURman, P.; Fraga, J. \& Martínez CORTIZAS, A. 2010. Holocene vegetation and hydrologic changes inferred from molecular vegetation markers in peat, Penido Vello (Galicia, Spain). Paleogeography, Peloclimatology, Palaeoecology 299, 56-69.

Silva-SÁnCHEZ, N.; LóPEZ-COSTAS, O. \& TALLÓNARMADA, R. 2013. Edafología, palinología y antropología física aplicadas a la arqueología ambiental. Estudos do Quaternário 9:1-14.

StUiver, M.; Reimer, P. 1993. Extended 14C database and revised CALIB radiocarbon calibration program. Radiocarbon. 35:215-230.

Urrutia, M.M.; García-Rodeja, E. \& Macías, F. 1989. Determinación del $\mathrm{pH}$ en suelos de carga variable de Galicia. Anales de Edafología y Agrobiología $48,219-228$ 